\section{Cultura de seguridad del paciente y factores asociados en una red de hospitales públicos españoles}

\author{
Patient safety culture and related factors in a \\ network of Spanish public hospitals \\ Cultura de segurança do paciente e fatores \\ associados em uma rede de hospitais públicos \\ espanhóis
}

Zenewton André da Silva Gama 1 Adriana Catarina de Souza Oliveira 2 Pedro Jesus Saturno Hernández 3

\title{
Resumen
}

1 Departamento de Saúde Coletiva, Universidade Federal do Rio Grande do Norte, Natal, Brasil.

2 Departamento de Enfermería, Universidad Católica San Antonio de Murcia, Murcia, España. ${ }^{3}$ UD. Medicina Preventiva y Salud Pública, Universidad de Murcia, Murcia, España.

Correspondencia Z. A. S. Gama Departamento de Saúde Coletiva, Universidade Federal do Rio Grande do Norte. Av. Gal. Cordeiro de Farias $s / n$, Natal, RN 59150-000, Brasil. zgama@ufrnet.br

\begin{abstract}
The objectives were to describe Patient Safety Culture (PSC) in a regional network of public hospitals, according to the perceptions of health professionals, and analyze the influence of socioprofessional factors. A survey was conducted with 1,113 professionals from eight Spanish hospitals, using a questionnaire that explores 12 dimensions of PSC. Perceptions were described through the Percentage of Positive (PPR) and Negative Responses (PNR) by dimension, and the association of factors was analyzed using multivariate linear regression. The dimension "Teamwork within the same Unit" had the highest PPR (73.5), and "Staffing" the highest PNR (61). The variables "Service" (Pharmacy, Surgical Center) and "Profession" (Nurses) were significantly associated to positive assessments. Thus, strategies to improve PSC should prioritize rational distribution of human resources, and take advantage of the positive perceived relationship within Units. Moreover, pharmaceutical and surgical services, as well as nurses should probably be benchmarked by other services and professionals.
\end{abstract}

Safety Management; Public Hospitals; Organizational Culture; Patient Safety
Este estudio objetivó describir la Cultura de Seguridad del Paciente (CSP) en una red de hospitales públicos, según las percepciones de los profesionales sanitarios, y analizar la asociación de factores sociolaborales. Se realizó una encuesta a 1.113 profesionales de ocho hospitales españoles, a través de un cuestionario con 12 dimensiones de la CSP. Las percepciones se describen mediante los Porcentajes de Respuestas Positivas (PRP) y Negativas (PRN) a cada dimensión, y se analiza la asociación de factores con regresión lineal múltiple. La dimensión "trabajo en equipo en la unidad" se destacó con el mayor PRP $(73,5)$ y “dotación de personal" con el mayor PRN (61). Los factores más asociados a la CSP $(p<0,05)$ fueron el "servicio" (farmacéutico y quirúrgico) y la "profesión" (enfermería), ambos de forma positiva. Así, la mejora de la CSP debe centrarse en la racionalidad de la dotación del personal y aprovecharse de la buena relación dentro de las unidades, utilizando los servicios farmacéutico y quirúrgico, y los enfermeros, como benchmark para otros servicios y profesionales.

Administración de la Seguridad; Hospitales Públicos; Cultura Organizacional; Seguridad del Paciente 


\section{Introducción}

El impacto de los resultados del informe To Err is Human ("Errar es humano") 1, publicado en 1999, puso en alerta las instituciones sanitarias en cuanto a la magnitud de los eventos adversos, generados por problemas relacionados con la falta de seguridad en dichas instituciones. Este informe reforzó con contundencia los hallazgos del "estudio Harvard", un trabajo pionero publicado por Brennan et al. 2, sobre la importancia de los eventos adversos derivados de una asistencia insegura, y fomentó la realización de otros estudios que identificaron el mismo problema en distintas partes del mundo 3,4,5,6.

Ante estos resultados, instituciones y organismos internacionales han considerado la seguridad del paciente un problema grave y de consecuencias con gran impacto. A partir de 2001, la seguridad es considerada por el Institute of Medicine (IOM) de Estados Unidos una de las seis dimensiones o atributos claves de la calidad de los servicios de salud 7. La definición más actual de seguridad, parecida, pero ampliada en relación a la del IOM, es aquella ofrecida por la Organización Mundial de la Salud (OMS), que considera la seguridad del paciente como " $a u$ sencia de daño, real o potencial, relacionado con los servicios de salud" 8 (p. 132).

Una de las reacciones más representativas fue de la propia OMS, que creó en 2004 una Alianza internacional para la seguridad del paciente 9 , hoy caracterizada como programa, que ha desarrollado desde entonces una serie de iniciativas importantes y globales. Adicionalmente, el National Quality Forum (NQF) de Estados Unidos 10 publicó en 2003 un conjunto de "prácticas seguras" que, debido a la evidencia disponible de su efectividad en la disminución de eventos adversos y su alta posibilidad de generalización, deberían ser aplicadas en los hospitales en general.

En este documento del NQF 10, la mejora de la cultura de seguridad del paciente ha sido señalada como la primera de las recomendaciones para fomentar la seguridad en hospitales, y dicha recomendación se reforzó en la reciente actualización de este documento en 2010 11. Se señala la medición de la cultura de seguridad, el feedback de los resultados, y el diseño de intervenciones consecuentes, como elementos necesarios para llevar a cabo esta recomendación. Tanto el NQF 10, como la OMS 9, consideran la cultura de seguridad como un indicador estructural básico que favorece la implementación de buenas prácticas clínicas e, incluso, la utilización efectiva de otras estrategias, como la notificación de incidentes y aprendizaje con los problemas.
Cultura de seguridad suele definirse como "el producto de los valores, actitudes, competencias y patrones de comportamiento individuales y colectivos que determinan el compromiso, el estilo y la capacidad de una organización de salud o de un sistema de seguridad" 12 (p. 23). Se entiende que dicha cultura es un constructo compuesto por dimensiones o subculturas, tales como el trabajo en equipo, comunicación, aprendizaje, etc. 13. La medición de la cultura de seguridad tiene como objetivo cuantificar las debilidades y fortalezas en cada subcultura, para favorecer las intervenciones de mejora, tanto de las percepciones y actitudes, como de los incidentes de seguridad 14 . Pese a que hay pocos estudios concluyentes sobre la relación entre cultura de seguridad deficiente, y la alta incidencia de errores o eventos adversos 15 , se sabe que este es un factor que contribuye a su ocurrencia 16 . Sus efectos positivos son evidentes al observar las mejoras en seguridad de otros sectores ajenos a los servicios de salud, como la industria química, la aviación o las plantas de energía nuclear, todos caracterizados por una fuerte cultura de seguridad 10,11. A pesar del amplio reconocimiento de su importancia, la medición del nivel de cultura de seguridad aún no es habitual en muchos ámbitos y poco se conoce de los factores asociados a este componente de la psicología de las organizaciones de salud y los aspectos claves que deben ser priorizados para su mejora.

En estos momentos, fruto de una anterior cooperación con el Ministerio de Sanidad y Consumo de España, disponemos de una herramienta válida y fiable, que adapta al contexto español la encuesta original de la Agency for Healthcare Research and Quality (AHRQ) y que puede ser utilizada para medir la cultura de seguridad del paciente en hospitales, identificar en qué habría que incidir para mejorarla, y poder monitorizar su evolución 17. Con estos antecedentes se realiza el presente estudio en una red de ocho hospitales públicos de una comunidad autónoma española (Región de Murcia), planteándose los siguientes objetivos: (i) describir la frecuencia de actitudes positivas y negativas, relacionadas con la seguridad del paciente, según los profesionales sanitarios de estos hospitales; (ii) analizar los factores sociolaborales y estructurales que están relacionados con la cultura de seguridad.

\section{Métodos}

El estudio se realiza en la red de hospitales públicos de la Región de Murcia (España) que prestan cuidados agudos a toda la población. El tipo de diseño utilizado fue observacional transversal. 
La recogida de datos se realizó mediante encuesta dirigida a los profesionales sanitarios de los hospitales. Se describe la cultura de seguridad en los hospitales, desde la perspectiva de los profesionales sanitarios, y se analiza la influencia de factores sociolaborales en esta percepción.

Participaron los ochos hospitales públicos de la Región. Uno de los hospitales es grande $(\geq 500$ camas), cuatro son de tamaño mediano (de 200 a 499 camas) y tres son pequeños ( $<200$ camas). En base al listado de personal de cada hospital, se seleccionaron profesionales sanitarios (médico, enfermero, farmacéutico, fisioterapeuta, psicólogo, bioquímico, etc.) con vínculo contractual fijo o interino adscritos a cualquiera de los servicios del hospital. Además, con el objetivo de lograr que el conocimiento de la realidad hospitalaria fuese lo más informado posible y minimizar el riesgo de incluir en la muestra a profesionales que ya no trabajasen en el centro, a la hora de distribuir el cuestionario, se han excluido los profesionales eventuales o que estaban realizando suplencias.

Las variables dependientes que reflejan el nivel de cultura de seguridad son la calificación global del grado de seguridad (pregunta única) y el porcentaje de respuestas o percepciones positivas, neutras y negativas a cada una de las dimensiones de la encuesta sobre cultura de seguridad. Las variables independientes, potencialmente predictoras del nivel de cultura de seguridad, son: años de trabajo en la profesión, años de trabajo en el hospital, años de trabajo en la unidad/servicio, horas de trabajo semanal, profesión (médico, enfermero, farmacéutico y otros), unidad o servicio (médico, quirúrgico, Unidad de Cuidados Intensivos - UCI, farmacia y otros) y tamaño del hospital donde trabaja (pequeño, mediano o grande).

El instrumento de medida utilizado fue el cuestionario Hospital Survey on Patient Safety Culture, cuyo proceso de traducción y validación a la lengua española está descrito en otro estudio 10. El cuestionario es de tipo autoadministrado e incluye 42 ítems relativos a la cultura de seguridad del paciente, agrupados en doce dimensiones; una pregunta sobre la calificación global del grado de seguridad percibido; y preguntas sobre características sociolaborales de los profesionales. Los 42 ítems relativos a las percepciones de los profesionales sobre la seguridad tienen cinco opciones de respuesta, construidas según una escala tipo Likert de 5 puntos [de muy en desacuerdo (1) a muy de acuerdo (5) ó de nunca (1) a siempre (5)]. Por otro lado, la pregunta de calificación global de grado de seguridad utiliza una escala unidimensional de 10 puntos [mínima seguridad (0) a máxima seguridad (10)].
Intentamos asegurar en torno a 100 encuestas contestadas por hospital (para una precisión de $\pm 0,01$ y un valor esperado de 0,5 , se necesitarían 96 encuestas). Así, considerando un porcentaje de respuesta esperado de entre el 30 y $50 \%$, se seleccionaron los sujetos necesarios mediante muestreo aleatorio estratificado por área del hospital (definidas en la sección A del cuestionario). En los hospitales pequeños (con plantillas de profesionales sanitarios inferiores a 300) se tomó el total del universo. Para aumentar la tasa de respuesta, se realizaron hasta dos recordatorios a los sujetos que no habían contestado. En total, se distribuyeron 3.141 cuestionarios en primera instancia.

Dado que con carácter general es necesario considerar el sesgo potencial de no respuesta para tasas de respuesta $<70 \%$, hemos analizado su importancia utilizando dos métodos: (a) comparando los resultados de las respuestas iniciales con los resultados de las respuestas a los recordatorios; y (b) ajustando la respuesta obtenida a las proporciones de las categorías en toda la muestra, en los casos en los que hay diferencias significativas en los resultados de las dimensiones entre estas categorías y tasas de respuesta diferentes, según estas mismas categorías. Para este ajuste, hemos considerado la variable profesión, puesto que influye significativamente y presenta tasas de respuesta también diferentes.

La calificación del grado de seguridad percibido se describe utilizando la media y los percentiles 25 y 75. Además, se describen los porcentajes de respuestas neutras, positivas y negativas en cada una de las dimensiones de la cultura de seguridad, acompañados de sus respectivos intervalos de confianza del 95\% (IC95\%), que sirven para clasificar las dimensiones en fortalezas o debilidades (oportunidades de mejora), tal como se explica más adelante.

Con el fin de guiar las actuaciones o intervenciones que pudiesen derivarse del estudio, de cara a mejorar la cultura de seguridad en los hospitales, el análisis por dimensiones se complementó con un gráfico de Pareto que especifica las dimensiones más problemáticas. Este gráfico aporta el porcentaje de respuestas negativas respecto a cada dimensión sobre el total de respuestas negativas ofrecidas al conjunto de ítems.

Los ítems del cuestionario con cinco opciones de respuesta fueron codificados en tres categorías: respuesta negativa, neutra y positiva. Esta codificación consideró que el cuestionario contiene tanto preguntas formuladas desde una perspectiva positiva como negativa (Tabla 1).

Además de posibilitar el cálculo de las frecuencias relativas de cada ítem, esta codificación permite medir indicadores compuestos de cada 
Codificación utilizada en el análisis de los datos en función del tipo de pregunta y opciones de respuesta del cuestionario.

\begin{tabular}{lccccc}
\hline $\begin{array}{l}\text { Formulación de la } \\
\text { pregunta }\end{array}$ & \multicolumn{2}{c}{ Negativa } & Codificación & & Neutra \\
& Muy en desacuerdo & En desacuerdo & Indiferente & De acuerdo & Muy de acuerdo \\
En positivo & Nunca & Raramente & A veces & Casi siempre & Siempre \\
En negativo & De acuerdo & Muy de acuerdo & Indiferente & Muy en desacuerdo & En desacuerdo \\
& Casi siempre & Siempre & A veces & Nunca & Raramente \\
\hline
\end{tabular}

dimensión, aplicando la siguiente fórmula para cada una de las tres categorías de respuesta:

$\Sigma$ respuestas (positivas, neutras o negativas) en los ítems de la dimensión x 100

Número de respuestas totales en los ítems de una dimensión

El resultado de esta operación sirve para clasificar las dimensiones como fortalezas o debilidades de la cultura de seguridad, al emplear los criterios utilizados y recomendados por la AHRQ: para las fortalezas, $\geq 75 \%$ de respuestas positivas ("de acuerdo/muy de acuerdo" ó "casi siempre/ siempre") a preguntas formuladas en positivo $\mathrm{y} \geq 75 \%$ de respuestas negativas ("en desacuerdo/muy en desacuerdo" ó "nunca/raramente”) a preguntas formuladas en negativo; y para las debilidades, $\geq 50 \%$ de respuestas negativas ("en desacuerdo/muy en desacuerdo" ó "raramente/ nunca") a preguntas formuladas en positivo $\mathrm{y} \geq$ $50 \%$ de respuestas positivas ("de acuerdo/muy de acuerdo" ó "casi siempre/siempre”) a preguntas formuladas en negativo 18 .

Se empleó regresión lineal múltiple (método stepwise) para analizar la influencia de los factores sociolaborales (profesión, tipo de servicio, años de profesión, etc.) en el porcentaje de respuestas positivas de cada dimensión y de todo el cuestionario, utilizando el coeficiente beta estandarizado, como resumen de la influencia de cada factor. Los análisis se realizaron con SPSS 15.0 (SPSS Inc., Chicago, Estados Unidos), considerando para las pruebas estadísticas como hipótesis nula la inexistencia de diferencias, que se rechaza cuando el nivel de significación es de $\mathrm{p}<0,05$.

El presente estudio se realizó dentro de las actividades de investigación del programa Evaluación y Mejora de la Calidad Asistencial (EMCA), mediante convenio entre la Conserjería de Sanidad de la Región de Murcia y la Universidad de Murcia.

\section{Resultados}

De los 3.141 cuestionarios enviados, un $35,4 \%$ respondió a la encuesta, variando en cada hospital de un mínimo de $20,8 \%$ a un máximo de $50 \%$. Aunque las tasas de respuestas son relativamente bajas, a pesar del recordatorio realizado, globalmente se alcanzó el objetivo numérico planteado y hemos podido contar con un total de 1.113 encuestas respondidas.

En el análisis comparativo de las respuestas iniciales con las respuestas a recordatorios (en teoría más próximos a la opinión de los que no responden), hemos encontrado diferencias significativas para cuatro dimensiones. Además, en dos dimensiones se aprecian diferencias en cuanto a la profesión de los encuestados. No obstante, al ajustar los promedios encontrados en función de un posible sesgo de no respuesta que introduciría este hecho, los valores con ajuste son muy parecidos con los no ajustados, con la mayoría de las diferencias en torno del $1 \%$, de forma que podemos considerar los resultados de la muestra obtenida como representativos de lo que hubiésemos obtenido con una tasa de respuesta mayor.

La mayoría de los profesionales que responde a la encuesta es personal de enfermería (62,3\%) y personal médico $(30,8 \%)$, estando bien representadas las tres profesiones más frecuentes (medicina, enfermería, farmacia), reflejando la importancia cuantitativa que tienen en los hospitales, aunque la tasa de respuesta es generalmente mayor en farmacéuticos y otros titulados (70\% y $48,4 \%$ respectivamente) que para medicina $(44,5 \%)$ o enfermería $(33,1 \%)$. Estas diferencias han sido tenidas en cuenta para el análisis y ajuste del sesgo de no respuesta explicado en la metodología del presente estudio. El 90,9\% de los que respondieron tiene contacto directo con los pacientes en su trabajo. De igual manera, la inmensa mayoría trabaja más de 20 horas a la semana en el hospital. Más del 70\% de los en- 
cuestados lleva trabajando más de 5 años en el hospital, y casi un tercio de éstos 21 años o más. Con alguna excepción, son una minoría en todos los hospitales (rango: 1,1-20,3\%; media: 5,7\%) los profesionales que llevan menos de un año en el servicio. La descripción completa de la muestra está reflejada en la Tabla 2.

En una escala de cero a diez, la calificación media es de 7,3 con un $25 \%$ de los casos que otorgaron una calificación por debajo de 6,5 y otro $25 \%$ por encima de 8,0 . Es decir, el $50 \%$ de los profesionales calificaron la seguridad en su servicio con una nota entre 6,5 y 8,0 .

La frecuencia de percepciones positivas del conjunto de profesionales para cada dimensión demostró que las dimensiones "trabajo en equipo en la misma unidad o servicio" y "expectativas y acciones de la dirección/supervisión de la unidad o servicio que favorecen la seguridad" presentaron los porcentajes de respuestas positivas más altos, respecto al total de posibles respuestas en las respectivas dimensiones, con un $73,5 \%$ (IC95\%: 71,6-75,4) y un 63,2\% (IC95\%: 61,0-65,4), respectivamente. No obstante, siguiendo los criterios de clasificación propuestos por la AHRQ, ninguna dimensión llega al nivel de fortaleza de la cultura de seguridad en el conjunto de hospitales (Tabla 3), es decir, un mínimo de $75 \%$ de respuestas positivas.

Por otro lado, algunas dimensiones presentaron un considerable porcentaje de respuestas negativas (Tabla 3). La dimensión "dotación de personal” presentó destacadamente el más alto porcentaje de percepción negativa, con un $61,0 \%$ (IC95\%: 59,1-62,8), llegando al nivel de debilidad de la cultura de seguridad u oportunidad de mejora, por superar el $50 \%$ de respuestas negativas. En el gráfico de Pareto (Figura 1), se aprecia que las oportunidades de mejora se centran sobre todo, y por este orden, en las dimensiones "dotación de personal”, "apoyo de la gerencia en la seguridad del paciente", "percepción de seguridad” y "trabajo en equipo entre unidades/servicios". Estas cuatro solas acumulan casi el $60 \%$ de todas las respuestas con carácter negativo.

De las 12 dimensiones estudiadas, solamente "percepción de seguridad" y "respuesta no punitiva a los errores" no tienen variables sociolaborales que influyen significativamente en su resultado, mostrándose así homogénea en cuanto a los factores independientes estudiados. En el resto de dimensiones, las variables que más se destacan como predictoras son el "servicio" y la "profesión". En cuanto a los servicios, "farmacia" y "servicio quirúrgico" se destacan en tres dimensiones cada uno, todas ellas en positivo. Por otra parte, la profesión "enfermería" aparece en el modelo predictivo de cinco dimensiones,

\section{Tabla 2}

Características de los profesionales encuestados en los ocho hospitales públicos de la Región de Murcia (España).

\begin{tabular}{|c|c|c|}
\hline Variables & $\mathrm{n}$ & $\%$ \\
\hline \multicolumn{3}{|c|}{ Contacto con el paciente } \\
\hline Sí & 1.012 & 92,3 \\
\hline No & 84 & 7,7 \\
\hline \multicolumn{3}{|c|}{ Años de profesión } \\
\hline Menos de 1 & 2 & 0,2 \\
\hline $1-5$ & 131 & 11,9 \\
\hline $6-10$ & 209 & 18,9 \\
\hline $11-15$ & 240 & 21,7 \\
\hline $16-20$ & 134 & 12,1 \\
\hline 21 o más & 369 & 33,4 \\
\hline \multicolumn{3}{|c|}{ Años en el hospital } \\
\hline Menos de 1 & 32 & 2,9 \\
\hline $1-5$ & 283 & 25,6 \\
\hline $6-10$ & 209 & 18,9 \\
\hline $11-15$ & 191 & 17,3 \\
\hline $16-20$ & 132 & 11,9 \\
\hline 21 o más & 241 & 21,8 \\
\hline \multicolumn{3}{|c|}{ Años en la unidad/servicio } \\
\hline Menos de 1 & 61 & 5,5 \\
\hline $1-5$ & 385 & 34,8 \\
\hline $6-10$ & 216 & 19,5 \\
\hline $11-15$ & 155 & 14,0 \\
\hline $16-20$ & 89 & 8,1 \\
\hline 21 o más & 153 & 13,8 \\
\hline \multicolumn{3}{|c|}{ Horas de trabajo semanal } \\
\hline Menos de 20 & 5 & 0,5 \\
\hline $20-39$ & 560 & 50,7 \\
\hline 40 o más & 478 & 43,3 \\
\hline \multicolumn{3}{|l|}{ Profesión } \\
\hline Enfermería & 497 & 45,0 \\
\hline Medicina & 273 & 24,7 \\
\hline Farmacia & 10 & 0,9 \\
\hline Otras & 15 & 1,4 \\
\hline \multicolumn{3}{|l|}{ Servicios } \\
\hline Médico & 281 & 25,4 \\
\hline Quirúrgico & 173 & 15,7 \\
\hline Farmacia & 4 & 0,4 \\
\hline $\mathrm{UCl}$ & 37 & 3,3 \\
\hline Otros & 239 & 21,6 \\
\hline \multicolumn{3}{|c|}{ Tamaño del hospital } \\
\hline Pequeño & 214 & 19,4 \\
\hline Mediano & 533 & 48,2 \\
\hline Grande & 358 & 32,4 \\
\hline
\end{tabular}


Tabla 3

Estimación puntual e intervalo de confianza del 95\% (IC95\%) del porcentaje de respuestas positivas, neutras y negativas en cada una de las dimensiones de la cultura de seguridad.

\begin{tabular}{lccc}
\hline Dimensión de la cultura de seguridad & $\begin{array}{c}\text { Positivas } \\
\%(\text { IC95\%) }\end{array}$ & $\begin{array}{c}\text { Neutras } \\
\%(\text { IC95\%) }\end{array}$ & $\begin{array}{c}\text { Negativas } \\
\%(\text { (IC95\%) }\end{array}$ \\
\hline 1. Frecuencia de eventos notificados & $44,8(42,3-47,4)$ & $36,0(33,8-38,3)$ & $19,1(17,2-21,1)$ \\
2. Percepción de seguridad & $48,7(46,8-50,6)$ & $18,2(16,9-19,5)$ & $33,1(31,5-34,8)$ \\
3. Acciones de la dirección/Supervisión que favorecen la seguridad & $63,2(60,1-65,4)$ & $21,3(19,7-22,9)$ & $15,5(14,0-17,1)$ \\
4. Aprendizaje organizacional/Mejora continua & $53,4(51,4-55,4)$ & $20,8(19,2-22,3)$ & $25,8(24,0-27,7)$ \\
5. Trabajo en equipo en la unidad/servicio & $73,5(71,6-75,4)$ & $12,8(11,5-14,0)$ & $13,7(12,2-15,2)$ \\
6. Franqueza en la comunicación & $49,4(47,3-51,5)$ & $32,9(31,1-34,7)$ & $17,7(16,2-19,3)$ \\
7. Feedback y comunicación sobre errores & $43,0(40,8-45,2)$ & $33,5(31,8-35,3)$ & $23,5(21,6-25,3)$ \\
8. Respuesta no punitiva a los errores & $52,5(50,4-54,5)$ & $20,4(18,8-21,9)$ & $27,1(25,4-28,9)$ \\
9. Dotación de personal & $28,2(26,5-29,9)$ & $10,8(9,8-11,8)$ & $61,0(59,1-62,8) *$ \\
10. Apoyo de la gerencia en seguridad del paciente & $28,9(26,6-31,1)$ & $29,7(27,8-31,6)$ & $41,4(39,2-43,7)$ \\
11. Trabajo en equipo entre unidad/servicio & $41,2(39,1-43,4)$ & $26,2(24,6-27,8)$ & $32,6(30,7-34,4)$ \\
12. Cambios de turno/Transiciones entre unidad/servicio & $50,8(48,6-52,9)$ & $20,5(19,0-22,0)$ & $28,8(26,9-30,7)$ \\
\hline
\end{tabular}

* Debilidad de la cultura de seguridad ( $\geq 50 \%$ de respuestas negativas).

Figura 1

Diagrama de Pareto enfocado en el porcentaje de respuestas negativas a las 12 dimensiones de la cultura de seguridad.

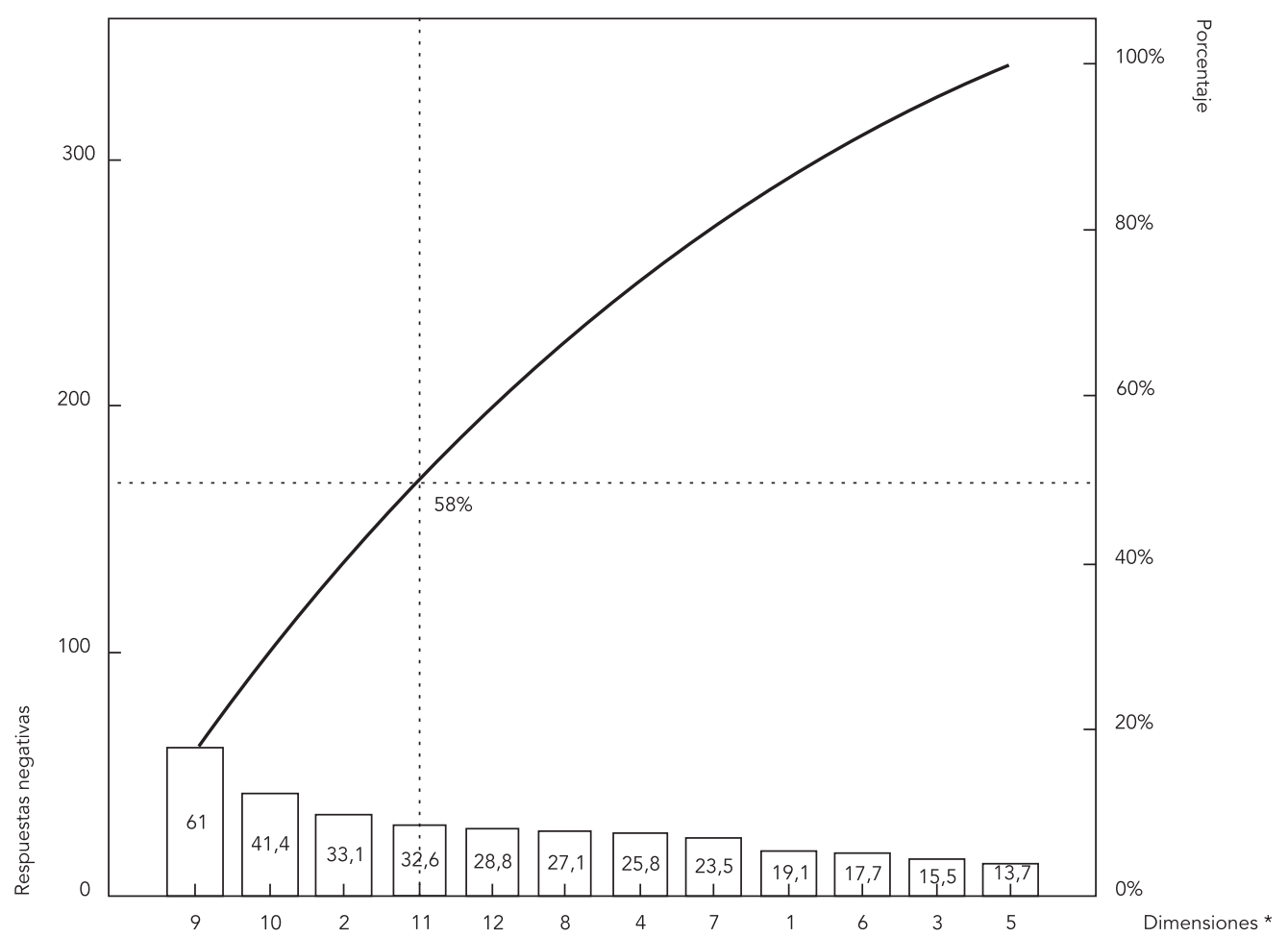

Nota: las barras representan el porcentaje medio de respuestas negativas en cada dimensión. La línea, el porcentaje acumulado de las respuestas negativas.

* El número identificador de cada dimensión es el mismo del utilizado en las Tablas 2 y 3. 
siempre presentando una relación directa con el porcentaje de respuestas positivas. Otra variable que se destaca en los modelos identificados es el "contacto directo con el paciente", ya que aparece en cuatro modelos, tres veces en positivo y una en negativo. La descripción completa de las variables sociolaborales que influyen en una percepción más positiva de las dimensiones de la cultura de seguridad puede verse en la Tabla 4.

\section{Discusión}

Este estudio aporta información sobre la cultura de seguridad en una muestra representativa de profesionales sanitarios que trabajan en hospitales públicos españoles. En general, se han identificado fortalezas y debilidades de la cultura de seguridad en estos hospitales, que deben servir para el diseño de intervenciones de mejora. Adicionalmente, se confirma la asociación significativa de algunos factores sociolaborales a la percepción positiva de la seguridad del paciente, tanto en general como por dimensiones.

A diferencia de otros estudios con el mismo instrumento de evaluación 18,19,20 que encuestaron profesionales sanitarios (médicos, enfermeros, etc.) y no sanitarios que trabajan en los hospitales (celadores, administrativos, etc.), hemos preferido restringir nuestra muestra a profesionales sanitarios con título universitario, teóricamente los que más conocen sobre los riesgos para la seguridad del paciente. Además, más del 90\% de los encuestados tenían contacto directo con el paciente. Esta característica de la muestra subraya la originalidad de este estudio, así como la identificación de factores asociados a la misma.

Es interesante destacar que los participantes consideran que su unidad/servicio tiene un grado de seguridad moderadamente alto (calificación 7,3), aunque mejorable. Esta percepción es mejor que la encontrada en otros estudios con la versión original del cuestionario que hemos utilizado 19,20, donde menos del 50\% de los participantes otorgaron una puntuación al grado de seguridad equivalente a nuestro "notable" $(7,0$ 8,9 puntos).

Aunque dos dimensiones destacan claramente en positivo, ninguna alcanza el nivel de "fortaleza de la cultura de seguridad" (estimación puntual: $\geq 75 \%$ de respuestas positivas), una clasificación "arbitraria" de los creadores de la encuesta 18. La dimensión "trabajo en equipo dentro de la unidad/servicio”, la más destacada en nuestro estudio $(73,5 \%$ de respuestas positivas; IC95\%: 71,6-75,4), también fue la más positiva en otros estudios tanto en España, como internacio- nalmente. Por ejemplo, esta dimensión alcanzó valores entre $70 \%$ y $80 \%$ de respuestas positivas en cuatro estudios independientes con el mismo cuestionario 19,20,21,22. Por otro lado, también se perciben como positivas las "acciones de la dirección/supervisión que favorecen la seguridad del paciente", quizás relacionada también con la buena percepción del trabajo en equipo dentro de la unidad. Según Wiegmann et al. 23, en su revisión sobre cultura de seguridad en la aviación y otras industrias del alto riesgo, esta dimensión puede ser clave al ejercer una fuerza positiva en la percepción de seguridad. La consistencia de sus resultados puede indicar la buena relación intra unidad/servicio y con sus directores o supervisores inmediatos, y puede ser aprovechada como un eficaz apoyo para la promoción de las intervenciones de mejora de la seguridad en este ámbito.

Respecto a las debilidades, se centran sobre todo y por este orden en las dimensiones "dotación de personal”, "apoyo de la gerencia en la seguridad de los pacientes", "percepción de seguridad" y "trabajo en equipo entre unidades/ servicios". Tal como se aprecia en el diagrama de Pareto (Figura 1), estas cuatro solas acumulan más del $50 \%$ de todas las respuestas con carácter negativo. De ellas, “dotación de personal” destaca claramente como debilidad, por superar los $50 \%$ de respuestas negativas. La razón puede ser la insatisfacción con las "condiciones de trabajo”, como la carga excesiva de trabajo, jornada laboral agotadora y trabajo bajo presión. Y aunque se puede argumentar que el personal siempre se queja de la carga de trabajo, no se puede ignorar esa percepción negativa cuando el profesional también apunta que la carga de trabajo excesiva se acompaña de un aumento de las respuestas negativas en la dimensión "percepción de seguridad”. Numerosos estudios 18,19,20,21,24 realizados en varios países también argumentan que la dotación de personal puede estar intrínsecamente relacionada con los incidentes de seguridad, evidenciando la importancia de prestar atención prioritaria a la racionalidad de la dotación y organización del personal y los ritmos de trabajo que se pueden ver sometidos, ya que en este ámbito es donde se percibe la existencia de debilidades y oportunidades de mejora. Los resultados negativos también evidencian una demonstración insuficiente por parte de la gerencia del hospital, en relación a sus actividades dirigidas a mejorar la seguridad del paciente, y aunque el trabajo en equipo dentro de la uni$\mathrm{dad} / \mathrm{servicio} \mathrm{es} \mathrm{casi} \mathrm{una} \mathrm{fortaleza,} \mathrm{se} \mathrm{perciben}$ fallos en la coordinación entre unidades/servicios distintos que repercuten negativamente en la seguridad. 
Resultados del análisis multivariante (regresión lineal múltiple) de la influencia de los factores sociolaborales y estructurales en la cultura de seguridad del paciente, cuantificada mediante el porcentaje de respuestas positivas a cada dimensión y al total de la encuesta.

\begin{tabular}{|c|c|c|c|c|}
\hline Dimensión de la cultura de seguridad & Factores significativos & $\begin{array}{l}\text { Coeficiente } \beta \\
\text { estandarizado }\end{array}$ & $\begin{array}{l}\text { Significación } \\
\text { del modelo }\end{array}$ & $\mathbf{R}^{2}$ \\
\hline \multirow[t]{3}{*}{ 1. Frecuencia de eventos notificados } & Profesión: enfermería & 0,078 & 0,000 & 0,019 \\
\hline & Hospitales pequeños & 0,077 & & \\
\hline & Años en profesión & 0,073 & & \\
\hline 2. Percepción de seguridad & - & - & $>0,05$ & - \\
\hline 3. Acciones de la dirección & Profesión: enfermería & 0,093 & 0,004 & 0,009 \\
\hline \multirow[t]{2}{*}{ 4. Aprendizaje organizacional } & Profesión: enfermería & 0,139 & 0,000 & 0,023 \\
\hline & Servicio de farmacia & 0,067 & & \\
\hline \multirow[t]{2}{*}{ 5. Trabajo en equipo en la unidad/servicio } & Contacto paciente & 0,118 & 0,000 & 0,019 \\
\hline & Servicio de quirófano & 0,067 & & \\
\hline \multirow[t]{2}{*}{ 6. Franqueza en la comunicación } & Contacto paciente & 0,100 & 0,002 & 0,013 \\
\hline & Servicio de farmacia & 0,077 & & \\
\hline \multirow[t]{4}{*}{ 7. Feedback y comunicación sobre errores } & Servicio de quirófano & 0,092 & 0,000 & 0,032 \\
\hline & Servicio de farmacia & 0,077 & & \\
\hline & Hospitales pequeños & 0,130 & & \\
\hline & Hospitales medianos & 0,103 & & \\
\hline 8. Respuesta no punitiva a los errores & - & - & $>0,05$ & - \\
\hline 9. Dotación de personal & Servicio otros & 0,088 & 0,007 & 0,008 \\
\hline 10. Apoyo de la gerencia en seguridad del & Horas trabajadas & $-0,099$ & 0,000 & 0,042 \\
\hline \multirow[t]{3}{*}{ paciente } & Contacto paciente & $-0,109$ & & \\
\hline & Profesión: enfermería & 0,107 & & \\
\hline & Profesión: farmacia & 0,068 & & \\
\hline 11. Trabajo en equipo entre unidad/servicio & Años en unidad/servicio & $-0,077$ & 0,018 & 0,006 \\
\hline 12. Cambios de turno y transiciones entre & Servicio de quirófano & 0,101 & 0,000 & 0,054 \\
\hline \multirow[t]{4}{*}{ unidad/servicio } & Horas trabajadas & $-0,096$ & & \\
\hline & Contacto paciente & 0,079 & & \\
\hline & Profesión: enfermería & 0,094 & & \\
\hline & Servicios otros & $-0,080$ & & \\
\hline \multirow[t]{6}{*}{ Todas las dimensiones (global) } & Horas trabajadas & $-0,070$ & 0,000 & 0,018 \\
\hline & Años en unidad/servicio & $-0,044$ & & \\
\hline & Servicio quirúrgico & 0,050 & & \\
\hline & Profesión: farmacia & 0,052 & & \\
\hline & Hospitales pequeños & 0,041 & & \\
\hline & Profesión: enfermería & 0,044 & & \\
\hline
\end{tabular}

Otro resultado importante es que los factores más asociados a la cultura de seguridad son el tipo de servicio y la profesión. En varias dimensiones se destacan positivamente el servicio de farmacia y quirófano. Posiblemente, este resultado sea fruto de la influencia de las muchas estrategias implantadas a nivel global por el programa de seguridad del paciente de la OMS 9, que ha priorizado las cirugías seguras 25 y el uso seguro de la medicación 26.
Por otro lado, los enfermeros se destacan positivamente en cinco de las 12 dimensiones, precisamente las relacionadas con el proceso de gestión de la seguridad del entorno laboral. Es decir, los enfermeros encuestados perciben que sus inmediatos superiores se preocupan por facilitar una cultura de seguridad favorable. Sin embargo, esta percepción positiva de los enfermeros no es muy consistente con los estudios realizados previamente. Aunque Pronovost et 
al. 27 encontraron que los enfermeros fueron los profesionales más destacados en la percepción de compromiso de la organización con la seguridad, otros estudios no apuntaron una percepción más positiva. Según Singer et al. ${ }^{28}$ y Kho et al. ${ }^{24}$, la profesión enfermería fue la característica que definió la percepción más pesimista en relación a la seguridad. Esta variabilidad puede deberse a situaciones realmente diferentes en los distintos contextos analizados o a diferencias relacionadas con los instrumentos o cuestionarios utilizados, que no eran los mismos aplicados en este estudio. El factor estructural "hospitales pequeños" aparece de forma significativamente positiva en dos dimensiones, y podría deberse a la menor dificultad de gestión en estos contextos.

En cuanto al instrumento, si bien es cierto que existen numerosas herramientas para medir la cultura de seguridad en el ámbito sanitario 18,24,28,29, el instrumento elegido tiene a su favor tres puntos esenciales: su extensa experiencia de aplicación (su estudio piloto contó con 382 hospitales 18, una actualización de los resultados en 2007 incluyó 519 hospitales 19, y una última actualización en 2012 que contó con la participación voluntaria de 1.128 hospitales) 20 ; su riguroso proceso de construcción y validación 18; y su fácil comprensión por parte de los participantes del estudio.

Respecto a la representatividad de la muestra, la utilización de muestreo aleatorio estratificado buscó evitar un posible sesgo de selección, pero el porcentaje de respuesta obtenido no llegó al $40 \%$, a pesar de las estrategias utilizadas para este objetivo. No obstante, el análisis a posteriori sobre un posible sesgo de no respuesta no reveló sesgo potencial en los resultados, mostrando que las estimaciones realizadas son representativas de las que podrían ser conseguidas con un porcentaje de respuesta más alto.
Por otro lado, a pesar de la consistencia de los resultados, y de la probable validez externa del estudio para contextos similares, hay que tener precaución al extrapolar nuestras conclusiones a otras redes de hospitales nacionales o de otros países. Las distintas realidades y la necesidad de intervenciones específicas no dispensan una medición individualizada, siguiendo la metodología reproducible presentada en este estudio, tanto para describir las dimensiones prioritarias de mejora, como los factores asociados. Según el consenso basado en la evidencia más actual 11, esta medición de la cultura de seguridad debería realizarse al menos anualmente, particularmente en las instituciones o sistemas de salud que ponen en marcha actividades concretas para intentar mejorarlo, atendiendo a las prioridades indicadas en la medición basal.

\section{Conclusiones}

A la vista de los resultados obtenidos en el ámbito de los hospitales estudiados, se puede concluir que se debe aprovechar la buena percepción de la interrelación interna en las diversas unidades/servicios, prestar atención a la racionalidad de la dotación de personal, fomentar la responsabilidad compartida y la coordinación entre unidades/servicios y mostrar una actitud más claramente proactiva hacia la seguridad del paciente por parte de la gerencia. Además, los servicios de farmacia y servicio quirúrgico, y la profesión de enfermería, constituyen factores fuertemente asociados a la percepción positiva de la cultura de seguridad, y deberían probablemente servir de benchmark para otros servicios y profesionales. 


\section{Resumo}

O estudo teve como objetivo descrever a Cultura de Segurança do Paciente (CSP) em uma rede de hospitais públicos, na percepção dos profissionais da saúde, e analisar a associação de fatores sociolaborais. Foi realizada uma pesquisa junto a 1.113 profissionais de oito hospitais espanhóis, através de um questionário com 12 dimensões da CSP. As percepções são descritas através dos porcentuais de respostas positivas (PRP) $e$ negativas (PRN), em cada dimensão, e é analisada a associação de fatores com regressão linear múltipla. A dimensão "trabalho em equipe na unidade" se destacou, com o maior PRP $(73,5)$ e "dotação em pessoal" com o maior PRN (61). Os fatores mais associados à $\operatorname{CSP}(p<0,05)$ foram o "serviço" (farmacêutico e cirúrgico) e a "profissão" (enfermaria), ambos de forma positiva. Dessa forma, a melhoria da CSP deve focar-se na racionalidade da dotação de pessoal e aproveitar a boa relação dentro das unidades, utilizando os serviços farmacêutico e cirúrgico, e os enfermeiros, como benchmark para os demais serviços e profissionais.

Gerenciamento de Segurança; Hospitais Públicos; Cultura Organizacional; Segurança do Paciente

\section{Colaboradores}

Z. A. S. Gama participó de la recogida de datos, análisis e interpretación de los datos; escribió el manuscrito y revisó su contenido; y aprobó la versión final enviada. A. C. S. Oliveira participó de la recogida de datos, análisis e interpretación de los datos; escribió el manuscrito y revisó su contenido; y aprobó la versión final enviada. P. J. Saturno Hernández contribuyó con el diseño el proyecto, y participó en el análisis e interpretación de los datos; escribió el manuscrito y revisó su contenido; y aprobó la versión final enviada.

\section{Agradecimientos}

Programa EMCA, Conserjería de Sanidad de la Región de Murcia (España).

\section{Referencias}

1. Institute of Medicine. To err is human: building a safer health system. Washington DC: National Academic Press; 1999.

2. Brennan TA, Leape LL, Laird NM, Hebert L, Localio AR, Lawthers AG, et al. Incidence of adverse events and negligence in hospitalized patients: results of the Harvard Medical Practice Study I. N Engl J Med 1991; 324:370-6.

3. Zegers M, Bruijne MC, Wagner C, Hoonhout LHF, Waaijman R, Smits M, et al. Adverse events and potentially preventable deaths in Dutch hospitals: results of a retrospective patient record review study. Qual Saf Health Care 2009; 18:297-302.

4. Aranaz-Andrés JM, Aibar-Remón C, Vitaller-Murillo J, Ruiz-López P, Limón-Ramírez R, Terol-García $\mathrm{E}$, et al. Incidence of adverse events related to health care in Spain: results of the Spanish National Study of Adverse Events. J Epidemiol Community Health 2008; 62:1022-9.

5. Mendes H, Martins M, Rozenfeld S, Travassos C. The assessment of adverse events in hospitals in Brazil. Int J Qual Health Care 2009; 21:279-84.
6. Aranaz-Andrés JM, Aibar-Remón C, Limón-Ramírez R, Amarilla A, Restrepo FR, Urroz O, et al. Prevalence of adverse events in the hospitals of five Latin American countries: results of the 'Iberoamerican study of adverse events' (IBEAS). BMJ Qual Saf 2011; 20:1043-51.

7. Institute of Medicine. Crossing the quality chasm. A new health system for the 21 century. Washington DC: National Academy Press; 2001.

8. World Health Organization. The conceptual framework for the International Classification for Patient Safety. Geneva: World Health Organization; 2009. (WHO Technical Report).

9. World Health Organization. World alliance for patient safety: forward program. Geneva:WHO Press; 2004.

10. The National Quality Forum. Safe practices for better healthcare: a consensus report. Washington DC: The National Quality Forum; 2003.

11. The National Quality Forum. Safe practices for better healthcare: 2010 update. Washington DC: The National Quality Forum; 2010. 
12. Health and Safety Commission Advisory Committee on the Safety of Nuclear Installations. Organizing for safety: third report of the ACSNI study group on human factors. Sudbury: HSE Books; 1993.

13. Sammer CE, Lykens K, Singh KP, Mains DA, Lackan NA. What is patient safety culture? A review of the literature. J Nurs Scholarsh 2010; 42:156-65.

14. Halligan M, Zecevic A. Safety culture in healthcare: a review of concepts, dimensions, measures and progress. BMJ Qual Saf 2011; 20:338-43.

15. Singer S, Lin S, Falwell A, Gama D, Baker L. Relationship of safety climate and safety performance in hospitals. Health Serv Res 2009; 44:399-421.

16. Pizzi LT, Goldfarb NI, Nash DB. Promoting a culture of safety. In: Making health care safer: a critical analysis of patient safety practices. Rockville: Agency for Healthcare Research and Quality; 2001. p. 447-57. (Evidence Report/Technology Assessment, 43/AHRQ Publication, 01-E058).

17. Gascón-Cánovas JJ, Saturno-Hernández PJ, PérezFernández P, Vicente-López I, González-Vicente A, Martínez-Mas J, et al. Cuestionario sobre seguridad de los pacientes: versión española del Hospital Survey on Patient Safety. Madrid: Ministerio de Sanidad y Consumo; 2005.

18. Sorra JS, Nieva VF. Hospital survey on patient safety culture. Rockville: Agency for Health Care Research and Quality; 2004. (AHRQ Publication, 04-0041).

19. Sorra J, Nieva V, Famolaro T, Dyer N. Hospital survey on patient safety culture 2007 comparative database report. Rockville: Agency for Healthcare Research and Quality; 2007. (AHRQ Publication, 08-0039).

20. Sorra J, Famolaro T, Dyer N, Nelson D, Smith SA. Hospital survey on patient safety culture 2012 user comparative database report. Rockville: Agency for Healthcare Research and Quality; 2012. (AHRQ Publication, 12-0017).

21. Hellings J, Schrooten W, Klazinga N, Vleugels A. Challenging patient safety culture: survey results. Int J Health Care Qual Assur 2007; 20:620-32.
22. Saturno PI, Da Silva-Gama ZA, De Oliveira-Sousa SL, Fonseca YA, De Souza-Oliveira AC. Analysis of the patient safety culture in hospitals of the Spanish national health system. Med Clín 2008; 131 Suppl 3:18-25.

23. Wiegmann DA, Zhang H, von Thaden T, Sharma G, Mitchell A. A synthesis of safety culture and safety climate research. Savoy: University of Illinois at Urbana-Champaign; 2002. (Technical Report ARL02-3/FAA-02-2).

24. Kho ME, Carbone JM, Lucas J, Cook DJ. Safety climate survey: reliability of results from a multicenter ICU survey. Qual Saf Health Care 2005; 14:273-8.

25. World Alliance for Patient Safety. Second global patient safety challenge: safe surgery saves lives. Geneva: WHO Press; 2008.

26. WHO Collaborating Center for Patient Safety Solutions, International Steering Committee. Patient safety solutions: preamble. Geneva: WHO Press; 2007.

27. Pronovost PJ, Weast B, Holsmuller C, Rosenstien B, Kidwel R, Haller K, et al. Evaluation of the culture of safety: survey of clinicians and managers in an academic medical center. Qual Saf Health Care 2003; 12:405-10.

28. Singer SJ, Gaba DM, Geppert JJ, Sinaiko AD, Howard SK, Park KC. The culture of safety: results of an organization: wide survey in 15 California hospitals. Qual Saf Health Care 2003; 12:112-8.

29. Sexton JB, Thomas EJ, Helmreich RL, Neilands TB, Rowan K, Vella K, et al. Frontline assessments of healthcare culture: safety attitudes questionnaire norms and psychometric properties. Austin: The University of Texas Center of Excellence for Patient Safety Research and Practice; 2004.

Recibido el 09/Ene/2012

Versión final presentada el 08/Sep/2012

Aprobado el 15/Oct/2012 